\title{
Follicular dendritic cell sarcoma of the spleen: A case report and review of the literature
}

\author{
LIFENG WANG $^{1}$, DONGFENG XU ${ }^{1}$, ZHENGUO QIAO ${ }^{2}$, LI SHEN $^{1}$, HONGZHI DAI ${ }^{1}$ and YIDING JI ${ }^{1}$ \\ Departments of ${ }^{1}$ Radiology and ${ }^{2}$ Gastroenterology, Affiliated Wujiang Hospital of Nantong University, \\ Suzhou, Jiangsu 215200, P.R. China
}

Received April 11, 2015; Accepted June 16, 2016

DOI: $10.3892 / \mathrm{ol} .2016 .4826$

\begin{abstract}
Follicular dendritic cell sarcoma (FDCS), which originates in antigen-presenting cells of the B-cell follicles of the lymph nodes, is an uncommon disease, particularly in a splenic location. In a number of cases, FDCS may easily be confused with other solid tumors and be underdiagnosed. The present study reports a case in which such as tumor occurred in a 64-year-old woman. The patient presented with an abdominal mass, and computed tomography was performed. Histological and immunohistochemical studies revealed a diagnosis of FDCS. The patient underwent surgical treatment and had a good remission. At the time of writing, the patient was in good condition. The present study also discusses the clinical characteristics, treatment methods and prognosis of FDCS, with particular reference to the main points of the imaging diagnosis.
\end{abstract}

\section{Introduction}

Follicular dendritic cells (FDCs), Langerhans cells and interdigitating dendritic cells are non-lymphoid, non-phagocytic cells classified generically as accessory cells of the lymphoid system. The function of non-phagocytic cells is the capture and presentation of antigens and immune complexes (1). Lymphoid follicles are present in extra-nodal lymphoid and lymph node tissues. As described by Monda et al, FDC sarcomas (FDSCs) were extremely rare in 1986 (2). In recent years, the incidence rates for FDSC has been gradually increasing. Recently, there have been a large number of reviews published on this neoplasm (3-5). Waldeyer's lymphoid tissue (tonsil) in the

Correspondence to: Mr. Zhenguo Qiao, Department of Gastroenterology, Affiliated Wujiang Hospital of Nantong University, 169 Gongyuan Road, Wujiang, Suzhou, Jiangsu 215200, P.R. China

E-mail: qzg66666666@163.com

Mr. Yiding Ji, Department of Radiology, Affiliated Wujiang Hospital of Nantong University, 169 Gongyuan Road, Wujiang, Suzhou, Jiangsu 215200, P.R. China

E-mail: 13405048687@163.com

Key words: follicular dendritic cells, pathology, diagnosis head and neck region is the most common extranodal site (40\%), followed by the nasopharynx $(20 \%)$, parapharyngeal space $(12 \%)$, maxillary alveolar ridge (4\%), and hard and soft palate (4\%) (6). The current study presents an additional case of FDCS of the spleen in a 64-year-old woman, and emphasizes the diagnostic difficulties in this specific population with a review of the pertinent literature.

\section{Case report}

In July 2013, an abdominal mass was found in a 64-year-old woman (who did not present obvious symptoms at that time) at a health center in Suzhou, China. The patient was then hospitalized in the Affiliated Wujiang Hospital of Nantong University (Suzhou, Jiangsu, China) for further examination by computed tomography (CT). Non-enhanced CT showed that this mass measured 190x124 mm, and was located in the spleen where a massive shadow was visible. The lesion manifested as a lower hybrid density shadow and calcification, with clear borders (Fig. 1). Moderate inhomogenous enhancement was found at the arterial phase (Fig. 2) and inhomogenous continuous enhancement was found at the venous phase (Fig. 3) following gadolinium administration, and a delayed CT scan showed that the lesion was significantly enhanced (Fig. 4).

A sample of the splenic mass was obtained at the time of the diagnostic evaluation and was formalin-fixed, paraffin-embedded, cut into $3-\mu \mathrm{m}$ sections, and stained with hematoxylin and eosin. The histological examination demonstrated that the diagnosis was splenic FDCS (Fig. 5).

The tissue sections were evaluated immunohistochemically using an avidin-biotin-peroxidase complex method and an automated immunostainer. All tissue sections were subjected to heat-induced antigen retrieval prior to incubation with mouse monoclonal anti-human antibodies against cluster of differentiation (CD)3 (sc-20047), CD20 (sc-393894), CD34 (sc-74499), CD68 (sc-20060), vimentin (sc-373717), neuron-specific enolase (NSE) (sc-376585), Ki-67 (sc-23900), CD15 (sc-19648), CD23 (sc-18910), CD30 (sc-46683) and epithelial membrane antigen (EMA) (sc-52329), which were purchased from Santa Cruz Biotechnology, Inc. (Dallas, TX, USA) and were used at a dilution 1:200. The tumor cells positively expressed CD3, CD20, CD34, CD68, vimentin and NSE. Ki-67 was expressed in 20\% of the tumor cell nuclei, and those tumor cells were negative for CD15, CD23, CD30 and EMA. In October 2013, in order 


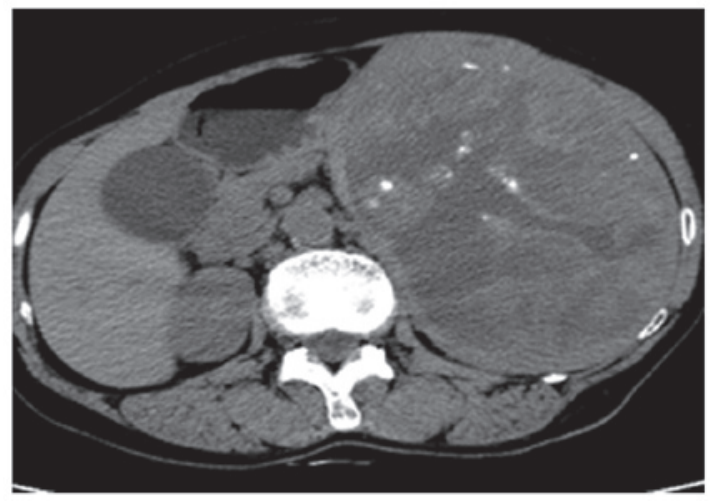

Figure 1. Non-enhanced computed tomography scan showing a 190x124-mm mass located in the spleen, where a massive shadow was visible. The lesion was manifested as a lower hybrid density shadow and calcification, with clear borders.

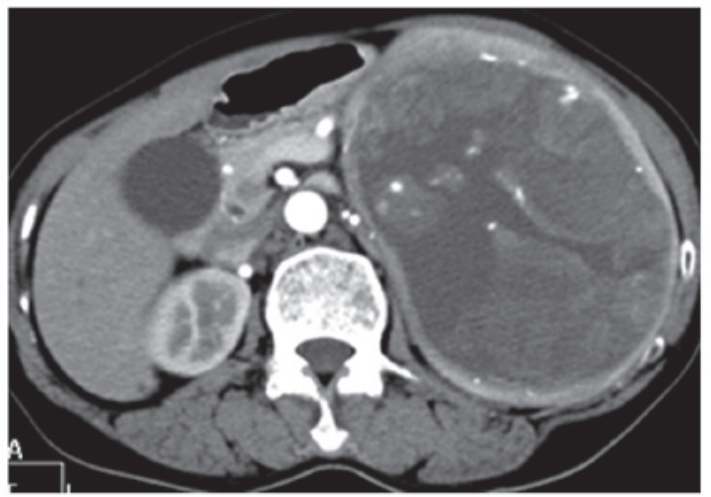

Figure 2. Computed tomography scan showing moderate inhomogenous enhancement at the arterial phase following gadolinium administration.

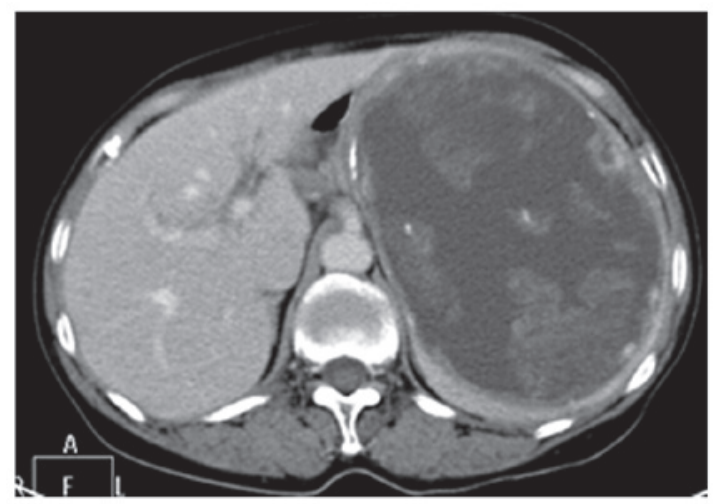

Figure 3. Computed tomography scan showing inhomogenous continuous enhancement at the venous phase following gadolinium administration.

to confirm the diagnosis established by the Affiliated Wujiang Hospital of Nantong University, the patient visited the Department of Pathology of the Affiliated Tumor Hospital of Fudan University (Shanghai, China) with the pathological sections and paraffin blocks for consultation. The result of the consultation was that the pathological diagnosis of the tumor accorded with splenic FDCS, which was consistent with the diagnosis made by the Affiliated Wujiang Hospital of Nantong University.

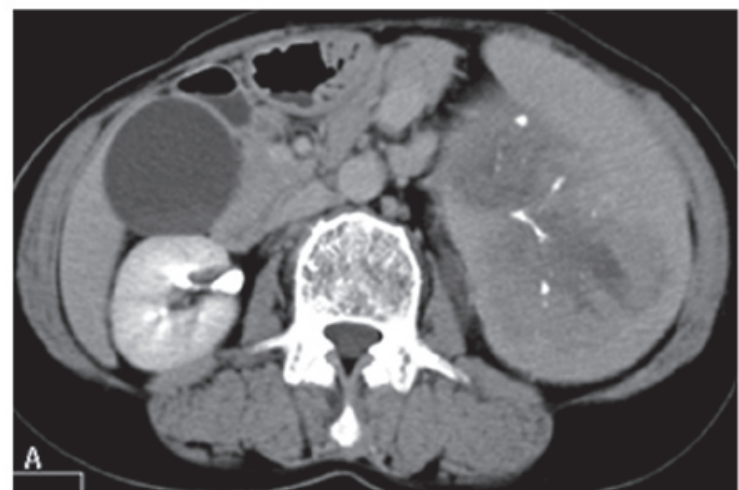

Figure 4. Delayed computed tomography scan showing the lesion with significant enhancement.

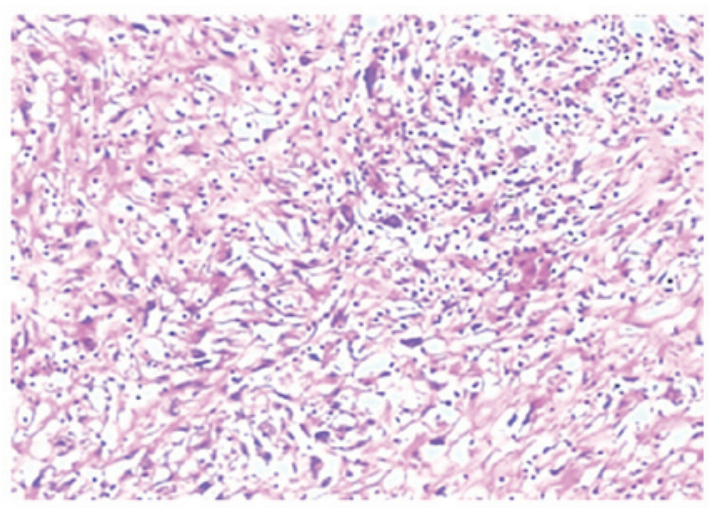

Figure 5. Morphological features of the atypical cells revealing large epithelioid cells with eosinophilic nucleoli and abundant cytoplasm (hematoxylin and eosin staining; magnification, x100).

Once the definitive diagnosis had been established, the patient received surgery in the Affiliated Wujiang Hospital of Nantong University. The patient had a good remission and not received chemotherapy or radiotherapy. At the time of writing, the patient was in a healthy condition. Written consent was obtained from the patient for publication of the present study.

\section{Discussion}

FDCS often occurs between the ages of 30 and 50, and there is no gender predilection (7). In the majority of cases, enlarged cervical lymph nodes are present, which are painless and slow growing (8). The patient in the present study only presented with an abdominal mass and did not present with any other obvious symptoms. The manifestations of the patient are similar to the cases reported in the literature $(9,10)$. Follicular dendritic cells are essential in the lymph nodes, where their primary role is directed towards antigen presentation and antigen-dependent B-cell maturation. Hence, the lymph nodes are the location in which this rare neoplasm most commonly occurs. However, the tumors can also colonize the inherent lymphoid tissues or be acquired by other organs that thereby serve as extranodal locations of occurrence (11). The histological features of FDCS tend to be stereotypical, with tumors composed of spindle or oval-shaped cells that show a storiform or whorled growth model, and are arranged in sheets, nets and fascicles focally (12). Epithelioid cells and 
multinucleated giant cells are visible in the lesions (13). These typical characteristics of pathology were in agreement with the results of the present case. Histological findings may increase the suspicion of FDCS, but it is essential to combine immunohistochemical staining for follicular cell differentiation in order to avoid misdiagnosis (14). CD21, CD23 and CD35 are the most widely used markers to demonstrate FDCS differentiation (15). Vimentin, EMA, S-100 protein, CD68, desmoplakin, muscle-specific actin and leukocyte common antigen show broadly variable positivity in FDCS. In fact, the only markers permitting the diagnosis of FDCS are CD21 and CD35 because they are only expressed by FDCS. Studies have shown that the pathogenesis of FDCS is associated with the Epstein-Barr (EB) virus, however, a review of the literature showed that EB virus RNA sequences were detected in the organs and tissues throughout the body, with the exception of the liver and spleen, in the majority of FDCS cases (16).

The majority of FDCS tumors have clear boundaries and are nodular or lobulated, with a size that is dependent on the location of the tumor. Usually, the tumors are small in volume, superficial and nodular with expansive growth, while hemorrhage or necrosis is rare. The tumors located in the abdominal cavity or abdominal organs are found deeper and are larger, with the presence of hemorrhage and necrosis, and possible infiltration into the adjacent parenchymal organs or soft tissues, as reported in the present case, which was consistent with the literature (17). On magnetic resonance imaging, FDCS may appear as an isointense expansive mass on T1-weighted scans, while homogeneous and slightly hyperintense on T2-weighted scans following gadolinium injection, with uniform moderate enhancement (18). These features may be useful in the diagnosis of this rare condition. However, more studies are required to describe the radiological characteristics of FDCS. As with any other sarcomas, a resection of the tumor with a wide margin is generally recommended whenever feasible. In cases with aggressive disease, such as those with extracapsular invasion, a high mitotic index or unresectable tumors, radiotherapy could be considered. Adjuvant chemotherapy, including a cyclophosphamide, hydroxydaunorubicin, oncovin and prednisone regimen, carboplatin or Adriamycin, has previously been recommended (19).

In conclusion, FDCS is a rare tumor that has been recognized only recently. The disease rate is probably underestimated, particularly when the tumors occur in extranodal sites. The present study describes an additional case of a woman with follicular dendritic cell sarcoma in the spleen, and discusses the main clinical characteristics, treatment options and prognosis of this neoplasm, particularly emphasizing the diagnostic difficulties. The aim of the present study is to aid radiologists and pathologists to further understand this tumor. Although the histological features of FDCS are rather stereotypical, they are likely to be misdiagnosed, as monoclonal-specific FDC markers are not routinely used in the immunohistochemical study of poorly-differentiated malignant tumors. The use of such markers appears to be necessary for any poorly-differentiated tumor. At present, surgical excision is the most effective treatment for the majority of patients with FDCS. In certain cases, where the tumor is deeply situated, large or infiltrated into the adjacent tissues, adjuvant chemotherapy and radiotherapy are the optimal treatments. Generally, the prognosis of FDCS is favorable $(18,20,21)$.

\section{References}

1. Karligkiotis A, Contis D, Bella M, Machouchas N, Volpi L, Melis A and Meloni F: Pediatric follicular dendritic cell sarcoma of the head and neck: A case report and review of the literature. Int J Pediatr Otorhinolaryngol 77: 1059-1064, 2013.

2. Monda L, Warnke R and Rosai J. A primary lymph node malignancy with features suggestive of dendritic reticulum cell differentiation. Am J Pathol 122: 562-572, 1986.

3. Vaideeswar P, George SM, Kane SV, Chaturvedi RA and Pandit SP: Extranodal follicular dendritic cell sarcoma of the tonsil-case report of an epithelioid cell variant with osteoclastic giant cells. Pathol Res Pract 205: 149-153, 2009.

4. Jiang L, Admirand JH, Moran C, Ford RJ and Bueso-Ramos CE: Mediastinal follicular dendritic cell sarcoma involving bone marrow: A case report and review of the literature. Ann Diagn Pathol 10: 357-362, 2006.

5. Romero-Guadarrama MB, Reyes-Posada O, HernándezGonzález MM and Durán-Padilla MA. Follicular dendritic cell sarcoma/tumor: 2 cases of a rare tumor of difficult clinical and pathological diagnosis. Ann Diagn Pathol 13: 257-262, 2009.

6. Chera BS, Orlando C, Villaret DB and Mendenahall WM: Follicular dendritic cell sarcoma of the head and neck: Case report and literature review. Laryngoscope 118: 1607-1612, 2008.

7. Tisch M, Hengstermann F, Kraft K, von Hinüber G and Maier H: Follicular dendritic cell sarcoma of the tonsil: Report of a rare case. Ear Nose Throat J 82: 507-509, 2003.

8. Hu T, Wang X, Yu C, Yan J, Zhang X, Li L, Li X, Zhang L, Wu J, Ma W, et al: Follicular dendritic cell sarcoma of the pharyngeal region. Oncol Lett 5: 1467-1476, 2013.

9. Li Z, Jin K, Yu X, Teng X, Zhou H, Wang Y, Teng L and Cao F: Extranodal follicular dendritic cell sarcoma in mesentery: A case report. Oncol Lett 2: 649-652, 2011.

10. Starr JS, Attia S, Joseph RW, Menke D, Casler J and Smallridge RC: Follicular dendritic cell sarcoma presenting as a thyroid mass. J Clin Oncol 33: e74-e76, 2015.

11. Perez-Ordoñez B and Rosai J: Follicular dendritic cell tumor: Review of the entity. Semin Diagn Pathol 15: 144-154, 1998.

12. Chan JK, Fletcher CD, Nayler SJ and Cooper K: Follicular dendritic cell sarcoma. Clinicopathologic analysis of 17 cases suggesting a malignant potential higher than currently recognized. Cancer 79: 294-313, 1997.

13. Ge R, Liu C, Yin X, Chen J, Zhou X, Huang C, Yu W and Shen X: Clinicopathologic characteristics of inflammatory pseudotumor-like follicular dendritic cell sarcoma. Int J Clin Exp Pathol 7: 2421-2429, 2014.

14. Biddle DA, Ro JY, Yoon GS, Yong YW, Ayala AG, Ordonez NG and Ro J: Extranodal follicular dendritic cell sarcoma of the head and neck region: Three new cases, with a review of the literature. Mod Pathol 15: 50-58, 2002.

15. Soriano AO, Thompson MA, Admirand JH, Fayad LE, Rodriguez AM, Romaguera JE, Hagemeister FB and Pro B: Follicular dendritic cell sarcoma: A report of 14 cases and a review of the literature. Am J Hematol 82: 725-728, 2007.

16. Shia J, Chen W, Tang LH, Carlson DL, Qin J, Guillem JG, Nobrega J, Wong WD and Klimstra DS: Extranodal follicular dendritic cell sarcoma: Clinical, pathologic, and histogenetic characteristics of an underrecognized disease entity. Virchows Arch 449: 148-158, 2006.

17. Encabo RS, McHugh J, Carrau RL, Kassam A and Heron D: Follicular dendritic cell sarcoma of the nasopharynx. Am J Otolaryngol 29: 262-264, 2008.

18. Clement P, Saint-Blancard P, Minvielle F, Le Page P and Kossowski M: Follicular dendritic cell sarcoma of the tonsil: A case report. Am J Otolaryngol 27: 207-210, 2006.

19. Fonseca R, Yamakawa M, Nakamura S, van Heerde P, Miettinen M, Shek TW, Myhre Jensen O, Rousselet MC and Tefferi A: Follicular dendritic cell sarcoma and interdigitating reticulum cell sarcoma: A review. Am J Hematol 59: 161-167, 1998.

20. Nakashima T, Kuratomi Y, Shiratsuchi H, Yamamoto $H$, Yasumatsu R, Yamamoto T and Komiyama S: Follicular dendritic cell sarcoma of the neck; a case report and literature review. Auris Nasus Larynx 29: 401-403, 2002.

21. Domínguez-Malagón H, Cano-Valdez AM, Mosqueda-Taylor A and Hes O. Follicular dendritic cell sarcoma of the pharyngeal region: Histologic, cytologic, immunohistochemical, and ultrastructural study of three cases. Ann Diagn Pathol 8: 325-332, 2004. 